\title{
Finite two-distance-transitive graphs of valency 6
}

\author{
Wei Jin *, Li Tan \\ School of Statistics, Jiangxi University of Finance and Economics, \\ Nanchang, Jiangxi, 330013, P.R.China \\ Research Center of Applied Statistics, Jiangxi University of Finance and Economics, \\ Nanchang, Jiangxi, 330013, P.R.China
}

Received 20 December 2014, accepted 8 April 2015, published online 18 August 2015

\begin{abstract}
A non-complete graph $\Gamma$ is said to be $(G, 2)$-distance-transitive if, for $i=1,2$ and for any two vertex pairs $\left(u_{1}, v_{1}\right)$ and $\left(u_{2}, v_{2}\right)$ with $d_{\Gamma}\left(u_{1}, v_{1}\right)=d_{\Gamma}\left(u_{2}, v_{2}\right)=i$, there exists $g \in G$ such that $\left(u_{1}, v_{1}\right)^{g}=\left(u_{2}, v_{2}\right)$. This paper classifies the family of $(G, 2)$-distancetransitive graphs of valency 6 which are not $(G, 2)$-arc-transitive.
\end{abstract}

Keywords: 2-Distance-transitive graph, 2-arc-transitive graph, permutation group.

Math. Subj. Class.: 05E18, 05B25

\section{Introduction}

In this paper, all graphs are finite, simple, connected and undirected. For a graph $\Gamma$, we use $V(\Gamma)$ and Aut $(\Gamma)$ to denote its vertex set and automorphism group, respectively. For the group theoretic terminology not defined here we refer the reader to $[4,8,26]$. Let $u, v \in V(\Gamma)$. Then the distance between $u, v$ in $\Gamma$ is denoted by $d_{\Gamma}(u, v)$. A non-complete graph $\Gamma$ is said to be $(G, 2)$-distance-transitive, if for $i=1,2$ and for any two vertex pairs $\left(u_{1}, v_{1}\right)$ and $\left(u_{2}, v_{2}\right)$ with $d_{\Gamma}\left(u_{1}, v_{1}\right)=d_{\Gamma}\left(u_{2}, v_{2}\right)=i$, there exists $g \in G$ such that $\left(u_{1}, v_{1}\right)^{g}=\left(u_{2}, v_{2}\right)$. An arc is an ordered pair of adjacent vertices. A vertex triple $(u, v, w)$ with $v$ adjacent to both $u$ and $w$ is called a 2-arc if $u \neq w$. The graph $\Gamma$ is said to be $(G, 2)$-arc-transitive if $G$ is transitive on both the set of arcs and the set of 2-arcs.

The first remarkable result about $(G, 2)$-arc-transitive graphs comes from Tutte [20, 21], and since then, this family of graphs has been studied extensively, see [1, 12, 15, 16, 17, $23,24]$. By definition, every non-complete $(G, 2)$-arc-transitive graph is $(G, 2)$-distancetransitive. The converse is not necessarily true. If a $(G, 2)$-distance-transitive graph has

\footnotetext{
* Supported by the NNSF of China (11301230), NSF of Jiangxi (20142BAB211008) and Jiangxi Education Department Grant (GJJ14351).

E-mail addresses: jinwei@jxufe.edu.cn (Wei Jin), tltanli@126.com (Li Tan)
} 
girth 3 (length of the shortest cycle is 3 ), then this graph is not $(G, 2)$-arc-transitive. Thus, the family of non-complete $(G, 2)$-arc-transitive graphs is properly contained in the family of $(G, 2)$-distance-transitive graphs. The graph in Figure 1 is the Kneser graph $K G_{6,2}$ which is $(G, 2)$-distance-transitive but not $(G, 2)$-arc-transitive of valency 6 for $G=\operatorname{Aut}\left(K G_{6,2}\right)$. Therefore the following problem naturally arises: characterize the family of $(G, 2)$-distance-transitive graphs. At the moment, Corr, Schneider and the first author are investigating such graphs, and they classified the family of $(G, 2)$-distance-transitive but not $(G, 2)$-arc-transitive graphs of valency at most 5 in [6]. Hence 6 is the next smallest valency for $(G, 2)$-distance-transitive graphs to investigate. Our main theorem gives a classification of such graphs.

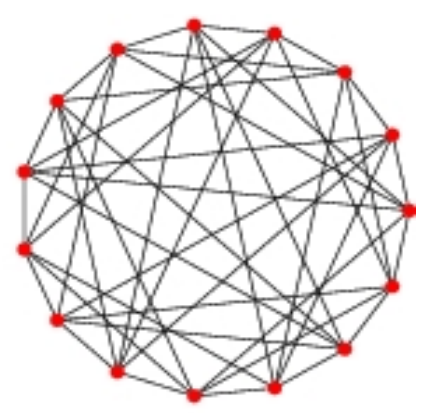

Figure 1: Kneser graph $K G_{6,2}$

Remark 1.1. Let $\Gamma$ be a connected $(G, 2)$-distance-transitive graph. If $\Gamma$ has girth at least 5 , then for any two vertices $u, v$ with $d_{\Gamma}(u, v)=2$, there exists a unique 2-arc between $u$ and $v$. Hence $\Gamma$ is $(G, 2)$-distance-transitive implies that it is $(G, 2)$-arc-transitive. If $\Gamma$ has girth 4 , then $\Gamma$ can be $(G, 2)$-distance-transitive but not $(G, 2)$-arc-transitive. There are infinitely many such graphs. For instance, let $\Gamma$ be the complement of the $\left(2 \times p^{k}\right)-$ grid where $p$ is a prime, and let $M=\mathbb{Z}_{p}^{k}: \mathbb{Z}_{p^{k}-1}, G=\mathbb{Z}_{2} \times M$. Then $\Gamma$ is $(G, 2)$-distance-transitive but not $(G, 2)$-arc-transitive of valency $p^{k}-1$ and girth 4 . There are also infinitely many $(G, 2)$-distance-transitive graphs of girth 4 that are $(G, 2)$-arc-transitive, for example the complete bipartite graphs $\mathrm{K}_{m, m}$. If $\Gamma$ has girth 3 , then since $\Gamma$ is non-complete, it follows that $G_{u}$ is not 2-transitive on $\Gamma(u)$, hence it is not $(G, 2)$-arc-transitive.

The line graph $L(\Gamma)$ of a graph $\Gamma$ has the set of edges of $\Gamma$ as its vertex set, and two edges are adjacent in $L(\Gamma)$ if and only if they have a common vertex in $\Gamma$. The line graph of a complete bipartite graph $\mathrm{K}_{m, n}$ is called an $(m \times n)$-grid. Let $\Gamma$ be a connected graph. The complement graph $\bar{\Gamma}$ of $\Gamma$, is the graph with vertex $V(\Gamma)$, and two vertices are adjacent in $\bar{\Gamma}$ if and only if they are not adjacent in $\Gamma$. The Hamming graph $\mathrm{H}(d, n)$ has vertex set $\mathbb{Z}_{n}^{d}=\mathbb{Z}_{n} \times \mathbb{Z}_{n} \times \cdots \times \mathbb{Z}_{n}$, and two vertices are adjacent if and only if they have exactly one different coordinate. We denote by $\mathrm{K}_{m[b]}$ the complete multipartite graph with $m$ parts, and each part has $b$ vertices where $m \geq 3, b \geq 2$. Let $p$ be a prime such that $p \equiv 1$ (mod 4). Then, the Paley graph $P(p)$ is the Cayley graph Cay $(T, S)$ for the additive group $T=F_{p}^{+}$with $S=\left\{w^{2}, w^{4}, \ldots, w^{p-1}=1\right\}$ and $\Gamma_{2}(1)=\left\{w, w^{3}, \ldots, w^{p-2}\right\}$, where $w$ is a primitive element of $F_{p}$, and $\operatorname{Aut}(\Gamma) \cong \mathbb{Z}_{p}: \mathbb{Z}_{\frac{p-1}{2}}$. In particular, Hamming graphs and Paley graphs are $(G, 2)$-distance-transitive for $G=\operatorname{Aut}(\Gamma)$, see [3, 13]. 
The diameter diam $(\Gamma)$ of a graph $\Gamma$ is the maximum distance occurring over all pairs of vertices. Let $u \in V(\Gamma)$ and $i=1,2, \ldots, \operatorname{diam}(\Gamma)$. We use $\Gamma_{i}(u)$ to denote the set of vertices at distance $i$ with vertex $u$ in $\Gamma$. Sometimes, $\Gamma_{1}(u)$ is also denoted by $\Gamma(u)$. Let $\Omega$ be a set of cardinality $n$. Then the Kneser graph $K G_{n, k}$ is the graph with vertex set all $k$-subsets of $\Omega$, and two $k$-subsets are adjacent if and only if they are disjoint. The triangular graph $T(n)$ is the graph with vertex set all 2-subsets of $\Omega$, and two 2-subsets are adjacent if and only if they share one common element. Thus $K G_{n, 2}=\overline{T(n)}$. A subgraph $X$ of $\Gamma$ is an induced subgraph if two vertices of $X$ are adjacent in $X$ if and only if they are adjacent in $\Gamma$. When $U \subseteq V(\Gamma)$, we use $[U]$ to denote the subgraph of $\Gamma$ induced by $U$.

Since complete graphs have diameter 1 , they do not provide interesting examples. Our main theorem determines the family of non-complete $(G, 2)$-distance-transitive graphs of valency 6 which are not $(G, 2)$-arc-transitive.

Theorem 1.2. Let $\Gamma$ be a connected non-complete $(G, 2)$-distance-transitive but not $(G, 2)$ arc-transitive graph of valency 6 . Let $u \in V(\Gamma)$. Then one of the following holds.

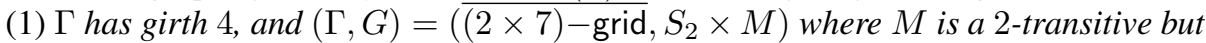
not 3-transitive subgroup of $S_{7}$.

(2) $[\Gamma(u)]$ is connected, and $\Gamma$ is isomorphic to one of: $T(5)$, Paley graph $P(13), K_{3[3]}$ or $\mathrm{K}_{4[2]}$.

(3) $[\Gamma(u)]$ is disconnected, and either

(3.1) $[\Gamma(u)] \cong 2 \mathrm{~K}_{3}, \Gamma \cong \mathrm{H}(2,4)$, or $\left|\Gamma_{2}(u)\right|=18$ and $\Gamma$ is a line graph; or

(3.2) $[\Gamma(u)] \cong 3 \mathrm{~K}_{2}, \Gamma \cong K G_{6,2}$, or $\left|\Gamma_{2}(u)\right|=12,24$.

Remark 1.3. (1) There exist graphs $\Gamma$ in Theorem 1.2 (3.1) such that $\left|\Gamma_{2}(u)\right|=18$. For instance the generalized hexagon of order $(3,1)$ and the generalized dodecagon of order $(3,1)$. These two graphs are locally isomorphic to $2 \mathrm{~K}_{3}$ and $\left|\Gamma_{2}(u)\right|=18$. By [3, p.223], they are $(G, 2)$-distance-transitive for $G=\operatorname{Aut}(\Gamma)$, since they are non-complete and have girth 3 , they are not $(G, 2)$-arc-transitive.

(2) There exist graphs $\Gamma$ in Theorem 1.2 (3.2) such that $\left|\Gamma_{2}(u)\right|=12$ and also exist graphs such that $\left|\Gamma_{2}(u)\right|=24$. For instance $\mathrm{H}(3,3)$ has valency $6,[\Gamma(u)] \cong 3 \mathrm{~K}_{2}$ and $\left|\Gamma_{2}(u)\right|=12$; the halved foster graph has valency $6,[\Gamma(u)] \cong 3 \mathrm{~K}_{2}$ and $\left|\Gamma_{2}(u)\right|=24$. By [3, p.223], these two graphs are $(G, 2)$-distance-transitive for $G=\operatorname{Aut}(\Gamma)$, since they are non-complete and have girth 3 , they are not $(G, 2)$-arc-transitive.

\section{Proof of Theorem 1.2}

In this section, we will prove our main theorem by a series of lemmas. All graphs are non-complete graphs.

A graph $\Gamma$ is said to be $G$-distance-transitive if $G$ is transitive on the ordered pairs of vertices at any given distance. The study of finite $G$-distance-transitive graphs goes back to Higman's paper [10] in which "groups of maximal diameter" were introduced. These are permutation groups $G$ which act distance-transitively on some graph. Then $G$-distancetransitive graphs have been studied extensively and a classification is almost done, see $[2,9,11,18,19,22,25]$. By definition, every non-complete $G$-distance-transitive graph is $(G, 2)$-distance-transitive.

The following remark gives an useful observation.

Remark 2.1. Let $\Gamma$ be a $(G, 2)$-distance-transitive graph. Let $u, w$ be two vertices such that $d_{\Gamma}(u, w)=2$. 
Suppose that $\left|\Gamma_{3}(u) \cap \Gamma(w)\right|=0$. Then since $\Gamma$ is $(G, 2)$-distance-transitive, $\Gamma$ has diameter 2 and so it is $G$-distance-transitive.

Suppose that $\left|\Gamma_{3}(u) \cap \Gamma(w)\right|=1$. Let $\left(u_{0}, \ldots, u_{i}\right)$ be a path with $d_{\Gamma}\left(u_{0}, u_{i}\right)=i$ where $i=\operatorname{diam}(\Gamma)$. Then for each $j \leq \operatorname{diam}(\Gamma)-2,\left|\Gamma_{3}\left(u_{j}\right) \cap \Gamma\left(u_{j+2}\right)\right|=1$. Note that, $\Gamma_{j+3}\left(u_{0}\right) \cap \Gamma\left(u_{j+2}\right) \subseteq \Gamma_{3}\left(u_{j}\right) \cap \Gamma\left(u_{j+2}\right)$, and so $\left|\Gamma_{j+3}\left(u_{0}\right) \cap \Gamma\left(u_{j+2}\right)\right|=1$, hence $\Gamma$ is also $G$-distance-transitive.

We use $G_{u}^{[1]}$ to denote the kernel of the $G_{u}$-action on $\Gamma(u)$.

Lemma 2.2. Let $\Gamma$ be a $(G, 2)$-distance-transitive graph. Let $u, w \in V(\Gamma)$ be such that $d_{\Gamma}(u, w)=2$. Let $g \in G_{u}^{[1]}$ be with order a prime $p$. Suppose that $\left|\Gamma_{3}(u) \cap \Gamma(w)\right|<p$. Then $g$ is not trivial on $\Gamma_{2}(u)$.

Proof. Suppose that $g$ is trivial on $\Gamma_{2}(u)$. Let $w_{i} \in \Gamma_{2}(u)$. Since $g \in G_{u}^{[1]}$ and $g$ is trivial on $\Gamma_{2}(u), g$ fixes all the vertices in $\left(\Gamma(u) \cup \Gamma_{2}(u)\right) \cap \Gamma\left(w_{i}\right)$ and $g \in G_{w_{i}}$. In particular, $g$ fixes $\Gamma_{3}(u) \cap \Gamma\left(w_{i}\right)$ setwise.

Since $\Gamma$ is $(G, 2)$-distance-transitive and $\left|\Gamma_{3}(u) \cap \Gamma(w)\right|<p,\left|\Gamma_{3}(u) \cap \Gamma\left(w_{i}\right)\right|<p$. Since the order of $g$ is prime $p$ and $g$ fixes $\Gamma_{3}(u) \cap \Gamma\left(w_{i}\right)$ setwise, it follows that $g$ fixes all the vertices in $\Gamma_{3}(u) \cap \Gamma\left(w_{i}\right)$. Thus $g \in G_{w_{i}}^{[1]}$. Since $w_{i}$ is any vertex of $\Gamma_{2}(u), g$ fixes all the vertices of $\Gamma_{3}(u)$. For any $v \in \Gamma(u), \Gamma_{2}(v) \subseteq \Gamma(u) \cup \Gamma_{2}(u) \cup \Gamma_{3}(u)$. Thus $g \in G_{v}^{[1]}$ and fixes all the vertices of $\Gamma_{2}(v)$.

Since $\Gamma$ is $(G, 2)$-distance-transitive, for any $z \in \Gamma_{2}(v),\left|\Gamma_{3}(v) \cap \Gamma(z)\right|<p$. Since $g$ fixes all the vertices in $\left(\Gamma(v) \cup \Gamma_{2}(v)\right) \cap \Gamma(z), g$ fixes all the vertices in $\Gamma_{3}(v) \cap \Gamma(z)$. Thus $g \in G_{z}^{[1]}$. In particular, $g$ fixes all the vertices of $\Gamma_{4}(u)$. Since $\Gamma$ is connected, by induction, $g$ fixes all the vertices of $\Gamma$, so $g=1$, which is a contradiction. Thus $g$ is not trivial on $\Gamma_{2}(u)$.

Lemma 2.3. Let $\Gamma$ be a $(G, 2)$-distance-transitive graph of valency 6 . Let $u, w \in V(\Gamma)$ be such that $d_{\Gamma}(u, w)=2$. If $\Gamma$ has girth 4 and $|\Gamma(u) \cap \Gamma(w)|=3$, then $\Gamma$ is $(G, 2)$-arctransitive.

Proof. Suppose that $\Gamma$ has girth 4 and $|\Gamma(u) \cap \Gamma(w)|=3$. Let $(u, v, w)$ be a 2 -arc. Then $d_{\Gamma}(u, w)=2$ and $\left|\Gamma_{2}(u) \cap \Gamma(v)\right|=5$. Since $\Gamma$ is $(G, 2)$-distance-transitive, there are 30 edges between $\Gamma(u)$ and $\Gamma_{2}(u)$. Since $|\Gamma(u) \cap \Gamma(w)|=3$ and $|\Gamma(u) \cap \Gamma(w)| \cdot\left|\Gamma_{2}(u)\right|=30$, it follows that $\left|\Gamma_{2}(u)\right|=10$. Again since $\Gamma$ is $(G, 2)$-distance-transitive, $G_{u}$ is transitive on both $\Gamma(u)$ and $\Gamma_{2}(u)$, so both $|\Gamma(u)|$ and $\left|\Gamma_{2}(u)\right|$ divide $\left|G_{u}\right|$, hence 30 divides $\left|G_{u}\right|$. Thus 5 divides $\left|G_{u, v}\right|$, so $G_{u, v}$ has an element $g$ of order 5 . Therefore either $\langle g\rangle$ is regular on $\Gamma(u) \backslash\{v\}$ or is trivial on $\Gamma(u) \backslash\{v\}$. If $\langle g\rangle$ is regular on $\Gamma(u) \backslash\{v\}$, then $G_{u, v}$ is transitive on $\Gamma(u) \backslash\{v\}$, so $G_{u}$ is 2-transitive on $\Gamma(u)$. Thus $\Gamma$ is $(G, 2)$-arc-transitive.

Now suppose that $g$ is trivial on $\Gamma(u) \backslash\{v\}$. Then $g \in G_{u}^{[1]}$. Since $|\Gamma(u) \cap \Gamma(w)|=3$, it follows that $\left|\Gamma_{3}(u) \cap \Gamma(w)\right| \leq 3<5$. Thus by Lemma 2.2, $g$ is not trivial on $\Gamma_{2}(u)$. Hence $\langle g\rangle$ has orbits of size 5 on $\Gamma_{2}(u)$. Since $g$ fixes $\Gamma_{2}(u) \cap \Gamma\left(v_{i}\right)$ setwise and $\left|\Gamma_{2}(u) \cap \Gamma\left(v_{i}\right)\right|=$ 5 , it follows that $\langle g\rangle$ is transitive on $\Gamma_{2}(u) \cap \Gamma\left(v_{i}\right)$. Thus $G_{u, v_{i}}$ is transitive on $\Gamma_{2}(u) \cap \Gamma\left(v_{i}\right)$, so $\Gamma$ is $(G, 2)$-arc-transitive.

Lemma 2.4. ([6]) Let $\Gamma \cong \mathrm{K}_{m, m}$ with $m \geq 2$. Then $\Gamma$ is $(G, 2)$-distance-transitive if and only if it is $(G, 2)$-arc-transitive.

A permutation group $G$ on a set $\Omega$ is said to be 2-homogeneous, if $G$ is transitive on the set of 2-subsets of $\Omega$. 
Lemma 2.5. ([8, Theorem 9.4B]) Let $G$ be a 2-homogeneous permutation group which is not 2-transitive of degree $n$. Then $n=p^{e} \equiv 3(\bmod 4)$ where $p$ is a prime.

Lemma 2.6. Let $\Gamma$ be a $(G, 2)$-distance-transitive but not $(G, 2)$-arc-transitive graph of

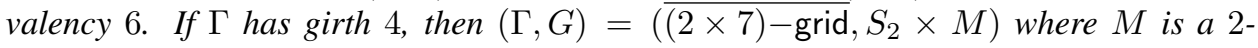
transitive but not 3-transitive subgroup of $S_{7}$.

Proof. Suppose that $\Gamma$ has girth 4. Let $(u, v, w)$ be a 2-arc. Then $d_{\Gamma}(u, w)=2, \mid \Gamma_{2}(u) \cap$ $\Gamma(v) \mid=5$ and $|\Gamma(u) \cap \Gamma(w)| \geq 2$. Further there are 30 edges between $\Gamma(u)$ and $\Gamma_{2}(u)$. Since $\Gamma$ is $(G, 2)$-distance-transitive, $|\Gamma(u) \cap \Gamma(w)|$ divides 30. Since $2 \leq|\Gamma(u) \cap \Gamma(w)| \leq$ 6 , we have $|\Gamma(u) \cap \Gamma(w)|=2,3,5$ or 6 .

Suppose first that $|\Gamma(u) \cap \Gamma(w)|=2$. Then since $\Gamma$ has girth 4, each 2-arc of $\Gamma$ lies in a unique 4-cycle. Thus, there is a 1-1 mapping between the unordered vertex pairs in $\Gamma(u)$ and vertices in $\Gamma_{2}(u)$. Since $G_{u}$ is transitive on $\Gamma_{2}(u)$, it follows that $G_{u}$ is transitive on the set of unordered vertex pairs in $\Gamma(u)$. Hence $G_{u}^{\Gamma(u)}$ is 2-homogeneous on $\Gamma(u)$. Further, since $\Gamma$ is not $(G, 2)$-arc-transitive, $G_{u}^{\Gamma(u)}$ is not 2-transitive on $\Gamma(u)$. Thus by Lemma 2.5, the valency of $\Gamma$ is $p^{e} \equiv 3(\bmod 4)$ where $p$ is a prime, contradicting the fact that $\Gamma$ has valency 6.

Next, if $|\Gamma(u) \cap \Gamma(w)|=3$, then by Lemma $2.3, \Gamma$ is $(G, 2)$-arc-transitive, which is a contradiction.

Thirdly, suppose that $|\Gamma(u) \cap \Gamma(w)|=5$. Then $\left|\Gamma_{3}(u) \cap \Gamma(w)\right| \leq 1$. It follows from Remark 2.1 that $\Gamma$ is $G$-distance-transitive. By inspecting the graphs in [3, p. 222-223],

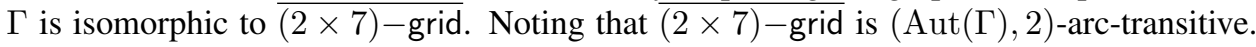
Thus $S_{2}<G<\operatorname{Aut}(\Gamma) \cong S_{2} \times S_{7}$. Let $G=S_{2} \times M$ where $M<S_{7}$. Then $G_{u}=M_{u}$. Since $\Gamma$ is $(G, 2)$-distance-transitive but not $(G, 2)$-arc-transitive, $M_{u}$ is transitive but not 2-transitive on $\Gamma(u)$. Thus $M$ is a 2-transitive but not 3-transitive subgroup of $S_{7}$.

Finally, if $|\Gamma(u) \cap \Gamma(w)|=6$, then $\Gamma \cong \mathrm{K}_{6,6}$, and by Lemma $2.4, \Gamma$ is $(G, 2)$-distancetransitive implies that it is $(G, 2)$-arc-transitive, which is a contradiction.

In a non-complete graph $\Gamma$, a 2-geodesic of $\Gamma$ is a 2-arc $\left(u_{0}, u_{1}, u_{2}\right)$ such that $d_{\Gamma}\left(u_{0}, u_{2}\right)$ $=2$. The graph $\Gamma$ is said to be $(G, 2)$-geodesic-transitive, if $G$ is transitive on both the set of arcs and the set of 2-geodesics. Hence, a non-complete $G$-arc-transitive graph is $(G, 2)$ geodesic-transitive if, for any $\operatorname{arc}(u, v), G_{u, v}$ is transitive on $\Gamma_{2}(u) \cap \Gamma(v)$. By definition, every $(G, 2)$-geodesic-transitive graph is $(G, 2)$-distance-transitive.

Suppose that $\Gamma$ is a $G$-distance-transitive graph of valency $k$ and diameter $d$. Then the cells of the distance partition with respect to vertex $u$ are orbits of $G_{u}$, every vertex in $\Gamma_{i}(u)$ is adjacent to the same number of other vertices in $\Gamma_{i-1}(u)$, say $c_{i}$. Similarly, every vertex in $\Gamma_{i}(u)$ is adjacent to the same number of other vertices in $\Gamma_{i+1}(u)$, say $b_{i}$. The notation $\left(k, b_{1}, \ldots, b_{d-1} ; 1, c_{2}, \ldots, c_{d}\right)$ is called the intersection array of $\Gamma$.

Lemma 2.7. Let $\Gamma$ be a $(G, 2)$-distance-transitive but not $(G, 2)$-arc-transitive graph of valency 6 . Let $u \in V(\Gamma)$. If $[\Gamma(u)]$ is connected, then $\Gamma$ is isomorphic to one of: $T(5)$, Paley graph $P(13), \mathrm{K}_{3[3]}$ or $\mathrm{K}_{4[2]}$.

Proof. Suppose that $[\Gamma(u)]$ is connected. Let $(u, v, w)$ be a 2 -arc such that $d_{\Gamma}(u, w)=$ 2. Since $\Gamma$ is $(G, 2)$-distance-transitive, $G_{u}$ is transitive on $\Gamma(u)$, so $[\Gamma(u)]$ is a vertextransitive graph. Let $k$ be the valency of $[\Gamma(u)]$. Since $[\Gamma(u)]$ is connected and $|\Gamma(u)|=6$, it follows that $k=2,3,4,5$. Let $\Gamma(u)=\left\{v_{1}, v_{2}, v_{3}, v_{4}, v_{5}, v_{6}\right\}$.

If $k=5$, then $[\Gamma(u)] \cong \mathrm{K}_{6}$, and so $\Gamma \cong \mathrm{K}_{7}$, contradicting the fact that $\Gamma$ is noncomplete. 
Suppose that $k=4$. Then $\left|\Gamma(u) \cap \Gamma\left(v_{1}\right)\right|=4$, say $\Gamma(u) \cap \Gamma\left(v_{1}\right)=\left\{v_{2}, v_{3}, v_{4}, v_{5}\right\}$. Since $\left|\Gamma(u) \cap \Gamma\left(v_{6}\right)\right|=4$ and $v_{1}, v_{6}$ are non-adjacent, it follows that $\Gamma(u) \cap \Gamma\left(v_{6}\right)=$ $\left\{v_{2}, v_{3}, v_{4}, v_{5}\right\}$. Thus $[\Gamma(u)]$ has diameter 2 , and $\left\{v_{1}, v_{6}\right\}$ is a block. Since $[\Gamma(u)]$ is vertex-transitive, $[\Gamma(u)] \cong \mathrm{K}_{3[2]}$, and by [3, p.5] or [5], $\Gamma \cong \mathrm{K}_{4[2]}$.

Suppose that $k=3$. Then $\left|\Gamma(u) \cap \Gamma\left(v_{1}\right)\right|=3$, say $\Gamma(u) \cap \Gamma\left(v_{1}\right)=\left\{v_{2}, v_{3}, v_{4}\right\}$. Assume first that $[\Gamma(u)]$ does not have triangles. Then every vertex of $\left\{v_{2}, v_{3}, v_{4}\right\}$ is adjacent to both $v_{5}$ and $v_{6}$. Thus $[\Gamma(u)] \cong \mathrm{K}_{3,3}$. Then by [3, p.5] or [5], $\Gamma \cong \mathrm{K}_{3[3]}$. Next, assume that $[\Gamma(u)]$ has a triangle. Since $[\Gamma(u)]$ is vertex-transitive, every vertex of $\Gamma(u)$ lies in a triangle. Let $\left(v_{1}, v_{2}, v_{3}\right)$ be a triangle. Since $[\Gamma(u)]$ is connected, $v_{4}$ is adjacent to neither $v_{2}$ nor $v_{3}$. Thus $v_{4}$ is adjacent to both $v_{5}$ and $v_{6}$. Since $v_{4}$ lies in a triangle and $\left\{v_{5}, v_{6}\right\} \subset \Gamma_{2}\left(v_{1}\right)$, it follows that $v_{5}, v_{6}$ are adjacent. Further, $v_{2}$ is adjacent to one of $\left\{v_{5}, v_{6}\right\}$, say $v_{5}$, and $v_{3}$ is adjacent to the remaining vertex $v_{6}$. Thus $[\Gamma(u)]$ is isomorphic to the 3 -prism, $\left(v_{1}, v_{2}, v_{3}\right)$ and $\left(v_{4}, v_{5}, v_{6}\right)$ are the two triangles, and $\left\{v_{1}, v_{4}\right\},\left\{v_{2}, v_{5}\right\}$ and $\left\{v_{3}, v_{6}\right\}$ are edges. Since $k=3$, it follows that $\left|\Gamma_{2}(u) \cap \Gamma\left(v_{1}\right)\right|=2$. Set $\Gamma_{2}(u) \cap \Gamma\left(v_{1}\right)=\left\{w_{1}, w_{2}\right\}$. Then $\Gamma\left(v_{1}\right)=\left\{u, v_{2}, v_{3}, v_{4}, w_{1}, w_{2}\right\}$. Since $\left[\Gamma\left(v_{1}\right)\right]$ is isomorphic to the 3-prism, it follows that $v_{4}$ is adjacent to both $w_{1}$ and $w_{2}, v_{2}$ is adjacent to one of $\left\{w_{1}, w_{2}\right\}$, say $w_{1}$, and $v_{3}$ is adjacent to $w_{2}$. Thus $\Gamma\left(v_{4}\right)=\left\{u, v_{1}, v_{5}, v_{6}, w_{1}, w_{2}\right\}$. Since $\left[\Gamma\left(v_{4}\right)\right]$ is isomorphic to the 3-prism, it follows that $w_{1}$ is adjacent to one of $\left\{v_{5}, v_{6}\right\}$, say $v_{5}$. Thus $\left\{v_{1}, v_{2}, v_{4}, v_{5}\right\} \subseteq$ $\Gamma(u) \cap \Gamma\left(w_{1}\right)$. Since $w_{2} \in \Gamma\left(w_{1}\right)$, it follows that $\left|\Gamma_{3}(u) \cap \Gamma\left(w_{1}\right)\right| \leq 1$. Thus by Remark 2.1, $\Gamma$ is $G$-distance-transitive.

Since $\left\{v_{1}, v_{2}, v_{4}, v_{5}\right\} \subseteq \Gamma(u) \cap \Gamma\left(w_{1}\right)$ and $\left\{w_{1}\right\} \subseteq \Gamma_{2}(u) \cap \Gamma\left(w_{1}\right)$, it follows that $\left|\Gamma(u) \cap \Gamma\left(w_{1}\right)\right|=4$ or 5 . Since $\Gamma$ is $(G, 2)$-distance-transitive and $\left|\Gamma_{2}(u) \cap \Gamma\left(v_{1}\right)\right|=2$, there are 12 edges between $\Gamma(u)$ and $\Gamma_{2}(u)$. Thus $\left|\Gamma(u) \cap \Gamma\left(w_{1}\right)\right|$ divides 12 , so $\mid \Gamma(u) \cap$ $\Gamma\left(w_{1}\right) \mid=4$. Hence $\left|\Gamma_{2}(u)\right|=3$. Since $G_{u}$ is transitive on $\Gamma_{2}(u),\left[\Gamma_{2}(u)\right]$ is a vertextransitive regular graph. Since $w_{1}, w_{2}$ are adjacent, $\left[\Gamma_{2}(u)\right] \cong C_{3}$. Therefore, $\mid \Gamma_{3}(u) \cap$ $\Gamma\left(w_{1}\right) \mid=0, \Gamma$ has diameter 2 and has 10 vertices. In particular, the intersection array of $\Gamma$ is $(6,2 ; 1,4)$. By inspecting the graphs in [3, p.222-223], $\Gamma$ is $T(5)$ (also known as the Johnson graph $J(5,2))$.

If $k=2$, then $[\Gamma(u)] \cong C_{6}$. Let $\left(v_{1}, \ldots, v_{6}\right)$ be a 6-cycle. Then $\left|\Gamma_{2}(u) \cap \Gamma\left(v_{1}\right)\right|=3$, and set $\Gamma_{2}(u) \cap \Gamma\left(v_{1}\right)=\left\{w_{1}, w_{2}, w_{3}\right\}$. Then $\Gamma\left(v_{1}\right)=\left\{u, v_{2}, v_{5}, w_{1}, w_{2}, w_{3}\right\}$. Since $\left[\Gamma\left(v_{1}\right)\right] \cong C_{6}$ and $\left(v_{2}, u, v_{6}\right)$ is a 2 -arc, it follows that $v_{2}$ is adjacent to one of $\left\{w_{1}, w_{2}, w_{3}\right\}$, say $w_{1} ; v_{6}$ is adjacent to one of $\left\{w_{2}, w_{3}\right\}$, say $w_{3}$; and $w_{2}$ is adjacent to both $w_{1}$ and $w_{3}$. In particular, $v_{2}$ is not adjacent to any of $\left\{w_{2}, w_{3}\right\}$, and $v_{6}$ is not adjacent to any of $\left\{w_{1}, w_{2}\right\}$. Since $\left|\Gamma_{2}(u) \cap \Gamma\left(v_{2}\right)\right|=3$, there exist $w_{4}, w_{5}$ in $\Gamma_{2}(u)$ that are adjacent to $v_{2}$, and so $\Gamma\left(v_{2}\right)=\left\{u, v_{1}, v_{3}, w_{1}, w_{4}, w_{5}\right\}$. Noting that $\left[\Gamma\left(v_{2}\right)\right] \cong C_{6}$ and $\left(w_{1}, v_{1}, u, v_{3}\right)$ is a 3 -arc, so $v_{3}$ is adjacent to one of $\left\{w_{4}, w_{5}\right\}$, say $w_{5}, w_{1}$ is adjacent to $w_{4}$, and $w_{4}, w_{5}$ are adjacent. Thus, $\left\{v_{1}, v_{2}, w_{2}, w_{4}\right\} \subseteq\left(\Gamma(u) \cup \Gamma_{2}(u)\right) \cap \Gamma\left(w_{1}\right)$. Hence $2 \leq\left|\Gamma(u) \cap \Gamma\left(w_{1}\right)\right| \leq 4$ and $\left|\Gamma_{2}(u) \cap \Gamma\left(w_{1}\right)\right| \geq 2$. Since $\Gamma$ is $(G, 2)$-distance-transitive and $\left|\Gamma_{2}(u) \cap \Gamma\left(v_{1}\right)\right|=3$, there are 18 edges between $\Gamma(u)$ and $\Gamma_{2}(u)$. Since $\left|\Gamma(u) \cap \Gamma\left(w_{1}\right)\right|$ divides $18,\left|\Gamma(u) \cap \Gamma\left(w_{1}\right)\right|=2$ or 3 .

Suppose that $\left|\Gamma(u) \cap \Gamma\left(w_{1}\right)\right|=2$. Then $\left|\Gamma_{2}(u)\right|=9$. Since $\left|\Gamma_{2}(u) \cap \Gamma\left(w_{1}\right)\right| \geq 2$, $\left|\Gamma_{3}(u) \cap \Gamma\left(w_{1}\right)\right| \leq 2$. If $\left|\Gamma_{3}(u) \cap \Gamma\left(w_{1}\right)\right| \leq 1$, then by Remark 2.1, $\Gamma$ is $G$-distancetransitive. Inspecting the graphs in [3, p. 222-223], such a $\Gamma$ does not exist. Hence $\mid \Gamma_{3}(u) \cap$ $\Gamma\left(w_{1}\right) \mid=2$. Since $\Gamma$ is $(G, 2)$-distance-transitive, both $|\Gamma(u)|$ and $\left|\Gamma_{2}(u)\right|$ divide $\left|G_{u}\right|$, hence 18 divides $\left|G_{u}\right|$. Thus 3 divides $\left|G_{u, v}\right|$. Therefore $G_{u, v}$ has an element $g$ of order 3 . Since $|\Gamma(u) \backslash\{v\}|=5$, it follows that $g$ is trivial on $\Gamma(u) \backslash\{v\}$, so $g \in G_{u}^{[1]}$. Hence $g$ fixes $\Gamma_{2}(u) \cap \Gamma\left(v_{i}\right)$ setwise. By Lemma 2.2,g is not trivial on $\Gamma_{2}(u)$. Hence $\langle g\rangle$ has orbits of 
size 3 on $\Gamma_{2}(u)$. Since $g$ fixes $\Gamma_{2}(u) \cap \Gamma\left(v_{i}\right)$ setwise and $\left|\Gamma_{2}(u) \cap \Gamma\left(v_{i}\right)\right|=3$, it follows that $\langle g\rangle$ is transitive on $\Gamma_{2}(u) \cap \Gamma\left(v_{i}\right)$. Thus $G_{u, v_{i}}$ is transitive on $\Gamma_{2}(u) \cap \Gamma\left(v_{i}\right)$. Therefore $\Gamma$ is $(G, 2)$-geodesic-transitive. Then by [7, Corollary 1.4], $\Gamma$ is either the Octahedron or the Icosahedron. However, these two graphs do not have valency 6 , which is a contradiction.

Finally, suppose that $\left|\Gamma(u) \cap \Gamma\left(w_{1}\right)\right|=3$. Since there are 18 edges between $\Gamma(u)$ and $\Gamma_{2}(u)$, and $\left|\Gamma_{2}(u)\right| \cdot\left|\Gamma(u) \cap \Gamma\left(w_{1}\right)\right|=18,\left|\Gamma_{2}(u)\right|=6$. Since $\left|\Gamma_{2}(u) \cap \Gamma\left(w_{1}\right)\right| \geq 2$, $\left|\Gamma_{3}(u) \cap \Gamma\left(w_{1}\right)\right| \leq 1$. Thus by Remark $2.1, \Gamma$ is $G$-distance-transitive. Inspecting the graphs in [3, p. 222-223], $\Gamma$ is the Paley graph $P(13)$.

Lemma 2.8. Let $\Gamma$ be a $(G, 2)$-distance-transitive graph of valency 6 . Let $u$ be a vertex of $\Gamma$. If $[\Gamma(u)] \cong 2 \mathrm{~K}_{3}$, then $\left|\Gamma_{2}(u)\right|=9$ or 18 .

Proof. Suppose that $[\Gamma(u)] \cong 2 \mathrm{~K}_{3}$. Then each arc lies in a unique $\mathrm{K}_{4}$. Let $\Gamma(u)=$ $\left\{v_{1}, v_{2}, v_{3}, v_{4}, v_{5}, v_{6}\right\}$ such that $\left(v_{1}, v_{2}, v_{3}\right)$ and $\left(v_{4}, v_{5}, v_{6}\right)$ are two triangles. Then for each $v_{i},\left|\Gamma_{2}(u) \cap \Gamma\left(v_{i}\right)\right|=3$. Since $\left[\Gamma\left(v_{1}\right)\right] \cong 2 K_{3}$, it follows that $\Gamma_{2}(u) \cap \Gamma\left(v_{i}\right) \cap \Gamma\left(v_{j}\right)=\emptyset$ for $i, j \in\{1,2,3\}$. Thus $\left|\Gamma_{2}(u)\right| \geq 9$.

On the other hand, since $\Gamma$ is $(G, 2)$-distance-transitive and $\left|\Gamma_{2}(u) \cap \Gamma\left(v_{1}\right)\right|=3$, there are 18 edges between $\Gamma(u)$ and $\Gamma_{2}(u)$. Thus $\left|\Gamma_{2}(u)\right|$ divides 18 , and so $\left|\Gamma_{2}(u)\right|=9$ or 18 .

If further $\left|\Gamma_{2}(u)\right|=9$, then such a graph is unique.

Lemma 2.9. Let $\Gamma$ be a $(G, 2)$-distance-transitive graph of valency 6 . Let $u$ be a vertex of $\Gamma$. Suppose that $[\Gamma(u)] \cong 2 \mathrm{~K}_{3}$ and $\left|\Gamma_{2}(u)\right|=9$. Then $\Gamma \cong \mathrm{H}(2,4)$

Proof. Since $[\Gamma(u)] \cong 2 \mathrm{~K}_{3}$, each arc lies in a unique $\mathrm{K}_{4}$. Let $\Gamma(u)=\left\{v_{1}, v_{2}, v_{3}, v_{4}, v_{5}\right.$, $\left.v_{6}\right\}$. Let $\left(v_{1}, v_{2}, v_{3}\right)$ and $\left(v_{4}, v_{5}, v_{6}\right)$ be the two triangles of $[\Gamma(u)]$. Then for each $v_{i}$, $\left|\Gamma_{2}(u) \cap \Gamma\left(v_{i}\right)\right|=3$. Since $\left[\Gamma\left(v_{1}\right)\right] \cong 2 \mathrm{~K}_{3}$, it follows that $\Gamma_{2}(u) \cap \Gamma\left(v_{i}\right) \cap \Gamma\left(v_{j}\right)=\emptyset$ for $i \neq j \in\{1,2,3\}$. Since $\left|\Gamma_{2}(u)\right|=9, \Gamma_{2}(u)=\left(\Gamma_{2}(u) \cap \Gamma\left(v_{1}\right)\right) \cup\left(\Gamma_{2}(u) \cap \Gamma\left(v_{2}\right)\right) \cup$ $\left(\Gamma_{2}(u) \cap \Gamma\left(v_{3}\right)\right)$. Set $\Gamma_{2}(u) \cap \Gamma\left(v_{1}\right)=\left\{w_{1}, w_{2}, w_{3}\right\}, \Gamma_{2}(u) \cap \Gamma\left(v_{2}\right)=\left\{w_{4}, w_{5}, w_{6}\right\}$, and $\Gamma_{2}(u) \cap \Gamma\left(v_{3}\right)=\left\{w_{7}, w_{8}, w_{9}\right\}$. Since $\left[\Gamma\left(v_{1}\right)\right] \cong\left[\Gamma\left(v_{2}\right)\right] \cong\left[\Gamma\left(v_{3}\right)\right] \cong 2 \mathrm{~K}_{3}$, it follows that $\left(w_{1}, w_{2}, w_{3}\right),\left(w_{4}, w_{5}, w_{6}\right)$ and $\left(w_{7}, w_{8}, w_{9}\right)$ are three triangles.

Since $\Gamma$ is $(G, 2)$-distance-transitive and $\left|\Gamma_{2}(u) \cap \Gamma\left(v_{1}\right)\right|=3$, there are 18 edges between $\Gamma(u)$ and $\Gamma_{2}(u)$. Since $\left|\Gamma_{2}(u)\right|=9$, it follows that for each $w_{i},\left|\Gamma(u) \cap \Gamma\left(w_{i}\right)\right|=2$. By the previous argument, $w_{1}$ is not adjacent to any of $\left\{v_{2}, v_{3}\right\}$, so $w_{1}$ is adjacent to one of $\left\{v_{4}, v_{5}, v_{6}\right\}$, say $v_{4}$. Then $\Gamma(u) \cap \Gamma\left(w_{1}\right)=\left\{v_{1}, v_{4}\right\}$. As each arc lies in a unique $\mathrm{K}_{4}$ and $\left(v_{1}, w_{1}, w_{2}, w_{3}\right)$ is a $\mathrm{K}_{4}$, it follows that $v_{4}$ is not adjacent to any of $\left\{w_{2}, w_{3}\right\}$. Since $\left|\Gamma_{2}(u) \cap \Gamma\left(v_{4}\right)\right|=3$ and $\left|\Gamma\left(v_{i}\right) \cap \Gamma\left(v_{4}\right)\right|=2$ for $i=1,2,3, v_{4}$ is adjacent to one of $\left\{w_{4}, w_{5}, w_{6}\right\}$, say $w_{4}$, and is adjacent to one of $\left\{w_{7}, w_{8}, w_{9}\right\}$, say $w_{7}$. Then $\Gamma\left(v_{4}\right)=\left\{u, v_{5}, v_{6}, w_{1}, w_{4}, w_{7}\right\}$. Since $\left[\Gamma\left(v_{4}\right)\right] \cong 2 \mathrm{~K}_{3}$ and $\left(u, v_{5}, v_{6}\right)$ is a triangle, it follows that $\left(w_{1}, w_{4}, w_{7}\right)$ is a triangle. Thus, $\Gamma\left(w_{1}\right)=\left\{v_{1}, v_{4}, w_{2}, w_{3}, w_{4}, w_{7}\right\}$, and so $\Gamma_{3}(u) \cap \Gamma\left(w_{1}\right)=\emptyset$. Since $\Gamma$ is $(G, 2)$-distance-transitive, it follows that $\Gamma$ is $G$-distancetransitive with diameter 2 and has 16 vertices. Thus by inspecting the graphs in [3, p. 222-223], $\Gamma \cong \mathrm{H}(2,4)$.

Lemma 2.10. Let $\Gamma$ be a $(G, 2)$-distance-transitive graph of valency 6 . Let $u$ be a vertex of $\Gamma$. If $[\Gamma(u)] \cong 3 \mathrm{~K}_{2}$, then $\left|\Gamma_{2}(u)\right|=8,12$, or 24 .

Proof. Suppose that $[\Gamma(u)] \cong 3 \mathrm{~K}_{2}$. Then each arc lies in a unique triangle. Let $\Gamma(u)=$ $\left\{v_{1}, v_{2}, v_{3}, v_{4}, v_{5}, v_{6}\right\}$ be such that $\left(v_{1}, v_{2}\right),\left(v_{3}, v_{4}\right)$, and $\left(v_{5}, v_{6}\right)$ are three arcs. Then for 
each $v_{i},\left|\Gamma_{2}(u) \cap \Gamma\left(v_{i}\right)\right|=4$. Since $\left[\Gamma\left(v_{1}\right)\right] \cong 3 \mathrm{~K}_{2}$, it follows that $\Gamma_{2}(u) \cap \Gamma\left(v_{1}\right) \cap \Gamma\left(v_{2}\right)=$ $\emptyset$. Thus $\left|\Gamma_{2}(u)\right| \geq 8$.

Since $\Gamma$ is $(G, 2)$-distance-transitive and $\left|\Gamma_{2}(u) \cap \Gamma\left(v_{1}\right)\right|=4$, there are 24 edges between $\Gamma(u)$ and $\Gamma_{2}(u)$. Since $\left|\Gamma_{2}(u)\right|$ divides 24, it follows that $\left|\Gamma_{2}(u)\right|=8,12$, or 24 .

If further $\left|\Gamma_{2}(u)\right|=8$, then $\Gamma$ is known.

Lemma 2.11. Let $\Gamma$ be a $(G, 2)$-distance-transitive graph of valency 6 . Let $u$ be a vertex of $\Gamma$. Suppose that $[\Gamma(u)] \cong 3 \mathrm{~K}_{2}$ and $\left|\Gamma_{2}(u)\right|=8$. Then $\Gamma \cong K G_{6,2}$

Proof. Since $\Gamma$ is symmetric and $[\Gamma(u)] \cong 3 \mathrm{~K}_{2}$, each arc lies in a unique triangle. Set $\Gamma(u)=\left\{v_{1}, v_{2}, v_{3}, v_{4}, v_{5}, v_{6}\right\}$. Let $\left(v_{1}, v_{2}\right),\left(v_{3}, v_{4}\right)$ and $\left(v_{5}, v_{6}\right)$ be three arcs. Then for each $v_{i},\left|\Gamma_{2}(u) \cap \Gamma\left(v_{i}\right)\right|=4$. Since $\left[\Gamma\left(v_{1}\right)\right] \cong 3 \mathrm{~K}_{2}$, it follows that $\Gamma_{2}(u) \cap \Gamma\left(v_{1}\right) \cap \Gamma\left(v_{2}\right)=$ $\emptyset$. Since $\left|\Gamma_{2}(u)\right|=8, \Gamma_{2}(u)=\left(\Gamma_{2}(u) \cap \Gamma\left(v_{1}\right)\right) \cup\left(\Gamma_{2}(u) \cap \Gamma\left(v_{2}\right)\right)$. Set $\Gamma_{2}(u) \cap \Gamma\left(v_{1}\right)=$ $\left\{w_{1}, w_{2}, w_{3}, w_{4}\right\}$, and $\Gamma_{2}(u) \cap \Gamma\left(v_{2}\right)=\left\{w_{5}, w_{6}, w_{7}, w_{8}\right\}$. Since $\left[\Gamma\left(v_{1}\right)\right] \cong\left[\Gamma\left(v_{2}\right)\right] \cong$ $3 \mathrm{~K}_{2}$, it follows that $\left(w_{1}, w_{2}\right),\left(w_{3}, w_{4}\right),\left(w_{5}, w_{6}\right)$ and $\left(w_{7}, w_{8}\right)$ are arcs.

Since $\Gamma$ is $(G, 2)$-distance-transitive and $\left|\Gamma_{2}(u) \cap \Gamma\left(v_{1}\right)\right|=4$, there are 24 edges between $\Gamma(u)$ and $\Gamma_{2}(u)$. As $\left|\Gamma_{2}(u)\right|=8$, it follows that for each $w_{i},\left|\Gamma(u) \cap \Gamma\left(w_{i}\right)\right|=3$. By the previous argument, $w_{1}$ is not adjacent to $v_{2}$. Noting that $\Gamma_{2}(u) \cap \Gamma\left(v_{i}\right) \cap \Gamma\left(v_{j}\right)=\emptyset$ for $(i, j)=(1,2),(3,4),(5,6)$. Thus $w_{1}$ is adjacent to one of $\left\{v_{3}, v_{4}\right\}$, say $v_{3}$, and is also adjacent to one of $\left\{v_{5}, v_{6}\right\}$, say $v_{5}$. Then $\Gamma(u) \cap \Gamma\left(w_{1}\right)=\left\{v_{1}, v_{3}, v_{5}\right\}$. Since each arc lies in a unique triangle and $\left(v_{1}, w_{1}, w_{2}\right)$ is a triangle, it follows that $v_{3}$ is not adjacent to $w_{2}$. By $\left|\Gamma_{2}(u) \cap \Gamma\left(v_{3}\right)\right|=4$ and $\left|\Gamma\left(v_{i}\right) \cap \Gamma\left(v_{3}\right)\right|=3$ for $i=1,2, v_{3}$ is adjacent to one of $\left\{w_{3}, w_{4}\right\}$, say $w_{3}$, and is also adjacent to two vertices of $\left\{w_{5}, w_{6}, w_{7}, w_{8}\right\}$, say $w_{5}, w_{7}$.

Then $\Gamma\left(v_{3}\right)=\left\{u, v_{4}, w_{1}, w_{3}, w_{5}, w_{7}\right\}$. Since $\left[\Gamma\left(v_{3}\right)\right] \cong 3 \mathrm{~K}_{2}$ and $\left(u, v_{4}\right)$ is an arc, it follows that $\left(w_{1}, w_{5}\right)$ and $\left(w_{3}, w_{7}\right)$ are two arcs. Thus, $\left\{v_{1}, v_{3}, v_{5}\right\} \cup\left\{w_{2}, w_{5}\right\} \subseteq$ $\Gamma\left(w_{1}\right)$, and so $\left|\Gamma_{3}(u) \cap \Gamma\left(w_{1}\right)\right| \leq 1$. Since $\Gamma$ is $(G, 2)$-distance-transitive, it follows from Remark 2.1 that $\Gamma$ is $G$-distance-transitive. One part of the intersection array of $\Gamma$ is $(6,4, \ldots ; 1,3, \ldots)$. By inspecting the graphs in $[3, \mathrm{p} .221], \Gamma \cong K G_{6,2}$.

Lemma 2.12. Let $\Gamma$ be an arc-transitive graph and let $u$ be a vertex of $\Gamma$. Suppose that $\Gamma(u)=U \cup W$, where $|U|=|W|=n$ and $U \cap W=\emptyset$. Assume further that $[U] \cong[W] \cong$ $\mathrm{K}_{n}$. Let $v_{1} \in U$. If $\left|\Gamma(u) \cap \Gamma\left(v_{1}\right) \cap W\right| \leq n-2$, then $\Gamma$ is a line graph.

Proof. Suppose that $\left|\Gamma(u) \cap \Gamma\left(v_{1}\right) \cap W\right| \leq n-2$. Then $[U]$ and $[W]$ are the only two $n$-cliques of $\Gamma(u)$. It follows from [14, Proposition 2.1] that $\Gamma$ is a line graph.

Proof of Theorem 1.2. Let $\Gamma$ be a connected non-complete $(G, 2)$-distance-transitive but not $(G, 2)$-arc-transitive graph of valency 6 . If $\Gamma$ has girth at least 5 , then for any two vertices with distance 2 , there exists a unique 2 -arc between these two vertices. Thus $\Gamma$ is $(G, 2)$-arc-transitive, which is a contradiction. Hence $\Gamma$ has girth 3 or 4 . If $\Gamma$ has girth 4 , then it follows from Lemma 2.6 that $(\Gamma, G)=\left(\overline{(2 \times 7) \text {-grid },} S_{2} \times M\right)$ where $M$ is a 2-transitive but not 3 -transitive subgroup of $S_{7}$, so that (1) holds.

Suppose that $\Gamma$ has girth 3 . Let $(u, v, w)$ be a 2 -arc such that $d_{\Gamma}(u, w)=2$. If $[\Gamma(u)]$ is connected, then by Lemma 2.7, $\Gamma$ is isomorphic to one of: $T(5)$, Paley graph $P(13), \mathrm{K}_{3[3]}$ or $\mathrm{K}_{4[2]}$, (2) holds. If $[\Gamma(u)]$ is disconnected, then $G_{u}$ has blocks in $\Gamma(u)$, and each block has cardinality 2 or 3 . If each block has cardinality 3 , then $[\Gamma(u)] \cong 2 \mathrm{~K}_{3}$; if each block has cardinality 2 , then $[\Gamma(u)] \cong 3 \mathrm{~K}_{2}$. Suppose that $[\Gamma(u)] \cong 2 \mathrm{~K}_{3}$. Then by Lemma 2.8 , $\left|\Gamma_{2}(u)\right|=9$ or 18 . If $\left|\Gamma_{2}(u)\right|=9$, then by Lemma $2.9, \Gamma \cong \mathrm{H}(2,4)$. If $\left|\Gamma_{2}(u)\right|=18$, then by Lemma 2.12, $\Gamma$ is a line graph, (3.1) holds. 
Finally, if $[\Gamma(u)] \cong 3 \mathrm{~K}_{2}$, then by Lemma $2.10,\left|\Gamma_{2}(u)\right|=8,12$, or 24 . In particular, if $\left|\Gamma_{2}(u)\right|=8$, then by Lemma $2.11, \Gamma \cong K G_{6,2}$, so that (3.2) holds.

\section{References}

[1] B. Alspach, M. Conder, D. Marušič and M. Y. Xu, A classification of 2-arc-transitive circulants, J. Algebraic Combin. 5 (1996), 83-86.

[2] E. Bannai and T. Ito, On distance regular graphs with fixed valency, Graphs and Combin. 3 (1987), 95-109.

[3] A. E. Brouwer, A. M. Cohen and A. Neumaier, Distance-Regular Graphs, Springer Verlag, Berlin, Heidelberg, New York, 1989.

[4] P. J. Cameron, Permutation Groups, volume 45 of London Mathematical Society Student Texts, Cambridge University Press, Cambridge, 1999.

[5] A. M. Cohen, Local recognition of graphs, buildings, and related geometries, edited by W. M. Kantor, R. A. Liebler, S. E. Payne and E. E. Shult, In Finite Geometries, Buildings, and related Topics, Oxford Sci. Publ., New York, 1990, 85-94.

[6] B. Corr, W. Jin and C. Schneider, Two-distance-transitive but not two-arc-transitive graphs, in preparation.

[7] A. Devillers, W. Jin, C. H. Li and C. E. Praeger, Line graphs and geodesic transitivity, Ars Math. Contemp. 6 (2013), 13-20.

[8] J. D. Dixon and B. Mortimer, Permutation groups, Springer, New York, 1996.

[9] A. Gardiner and C. E. Praeger, Distance transitive graphs of valency six, Ars Combin. A 21 (1986), 195-210.

[10] D. G. Higman, Intersection matrices for finite permutation groups, J. Algebra 6 (1967), 22-42.

[11] A. A. Ivanov and A. V. Ivanov, Distance transitive graphs of valency $k, 8 \leq k \leq 13$, in Algebraic, Extremal and Metric Combinatorics, in 1986, Cambridge Univ. Press, Cambridge, (1988), 112-145.

[12] A. A. Ivanov and C. E. Praeger, On finite affine 2-arc transitive graphs. European J. Combin. 14 (1993), 421-444.

[13] W. Jin, A. Devillers, C. H. Li and C. E. Praeger, On geodesic transitive graphs, Discrete Math., 338 (2015), 168-173.

[14] W. Jin, W. J. Liu and S. J. Xu, Line graphs and 2-geodesic transitive graphs, submitted.

[15] C. H. Li and J. M. Pan, Finite 2-arc-transitive abelian Cayley graphs, European J. Combin. 29 (2008), 148-158.

[16] D. Marušič, On 2-arc-transitivity of Cayley graphs, J. Combin. Theory Ser. B 87 (2003), 162196.

[17] C. E. Praeger, On a reduction theorem for finite, bipartite, 2-arc transitive graphs, Australas. J. Combin. 7 (1993) 21-36.

[18] C. E. Praeger, J. Saxl and K. Yokohama, Distance transitive graphs and finite simple groups, Proc. London Math. Soc. (3)55 (1987), 1-21.

[19] D. H. Smith, Distance transitive graphs of valency four, J. London Math. Soc. (2)8 (1974), 377-384.

[20] W. T. Tutte, A family of cubical graphs, Proc. Cambridge Philos. Soc. 43 (1947), 459-474.

[21] W. T. Tutte, On the symmetry of cubic graphs, Canad. J. Math. 11 (1959), 621-624. 
[22] J. Van Bon, Affine distance transitive groups, Proc. London Math. Soc., (3)67 (1993), 1-52.

[23] R. Weiss, $s$-Arc transitive graphs, Algebraic Metheods in Graph Theory I, II, (Szeged, 1978), Colloq. Math. Soc. Janos. Bolyai vol.25, North Holland, Amsterdam, 1978, 827-847.

[24] R. Weiss, The non-existence of 8-transitive graphs, Combinatorica 1 (1981), 309-311.

[25] R. Weiss, Distance transitive graphs and generalized polygons, Arch. Math. 45 (1985), 186192.

[26] H. Wielandt, Finite Permutation Groups, New York: Academic Press, 1964. 